\title{
Comparisons of the Pentax-AWS, Glidescope, and Macintosh Laryngoscopes for Intubation Performance during Mechanical Chest Compressions in Left Lateral Tilt: A Randomized Simulation Study of Maternal Cardiopulmonary Resuscitation
}

\author{
Sanghyun Lee, ${ }^{1}$ Wonhee Kim, ${ }^{2}$ Hyunggoo Kang, ${ }^{1}$ Jaehoon Oh, ${ }^{1}$ Tae Ho Lim, \\ Yoonjae Lee, ${ }^{1}$ Changsun Kim, ${ }^{1}$ and Jun Hwi $\mathrm{Cho}^{3}$ \\ ${ }^{1}$ Department of Emergency Medicine, College of Medicine, Hanyang University, Seoul 133-792, Republic of Korea \\ ${ }^{2}$ Department of Emergency Medicine, College of Medicine, Hallym University, Seoul, Republic of Korea \\ ${ }^{3}$ Department of Emergency Medicine, Kangwon National University School of Medicine, Chuncheon, Republic of Korea
}

Correspondence should be addressed to Hyunggoo Kang; emer0905@gmail.com

Received 11 November 2014; Accepted 25 December 2014

Academic Editor: Massimiliano Carassiti

Copyright (C) 2015 Sanghyun Lee et al. This is an open access article distributed under the Creative Commons Attribution License, which permits unrestricted use, distribution, and reproduction in any medium, provided the original work is properly cited.

Purpose. Rapid advanced airway management is important in maternal cardiopulmonary resuscitation (CPR). This study aimed to compare intubation performances among Pentax-AWS (AWS), Glidescope (GVL), and Macintosh laryngoscope (MCL) during mechanical chest compression in $15^{\circ}$ and $30^{\circ}$ left lateral tilt. Methods. In 19 emergency physicians, a prospective randomized crossover study was conducted to examine the three laryngoscopes. Primary outcomes were the intubation time and the success rate for intubation. Results. The median intubation time using AWS was shorter than that of GVL and MCL in both tilt degrees. The time to visualize the glottic view in GVL and AWS was significantly lower than that of MCL (all $P<0.05$ ), whereas there was no significant difference between the two video laryngoscopes (in $15^{\circ}$ tilt, $P=1$; in $30^{\circ}$ tilt, $P=0.71$ ). The progression of tracheal tube using AWS was faster than that of MCL and GVL in both degrees (all $P<0.001$ ). Intubations using AWS and GVL showed higher success rate than that of Macintosh laryngoscopes. Conclusions. The AWS could be an appropriate laryngoscope for airway management of pregnant women in tilt CPR considering intubation time and success rate.

\section{Introduction}

Emergency physicians are primarily responsible for both high-quality chest compressions and rapid advanced airway management in cardiopulmonary resuscitation (CPR) for women who are both pregnant and nonpregnant. The 2010 European Resuscitation Council (ERC) guidelines recommend employing manual displacement of the uterus with left lateral tilt (LLT) to achieve aortocaval decompression during CPR in late pregnancy [1]. High-quality chest compressions could be performed in the LLT position, which is not flat in simulated manikin studies [2]. Mechanical chest compression devices could compress the chest sufficiently regardless of the tilt $[2,3]$. Intubation time and the success rate for intubation are influenced by manual chest compressions and the type of laryngoscope [4-6]. The LLT position could also interfere with rapid intubation during chest compressions in the operating setting $[7,8]$. Physiological changes in a pregnant woman's airway (i.e., tongue swelling and airway edema) make endotracheal intubation more difficult [9-11]. Decreased functional residual capacity and increased risk of aspiration in a pregnant woman require rapid endotracheal intubation during CPR [12-14].

The optimal degree of LLT is not known. However, ERC guidelines recommend it be between $15^{\circ}$ and $30^{\circ}$ [1]. In an emergency room or delivery room, where maternal cardiac arrest commonly occurs, several methods are available to achieve the LLT position, whereas the operating table is used 


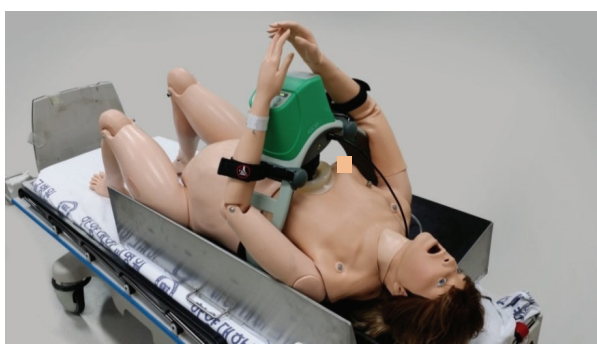

(a)

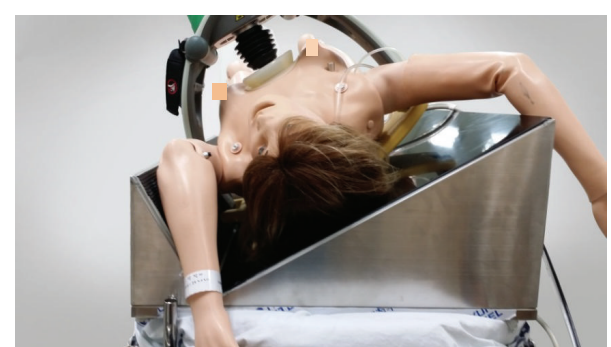

(b)

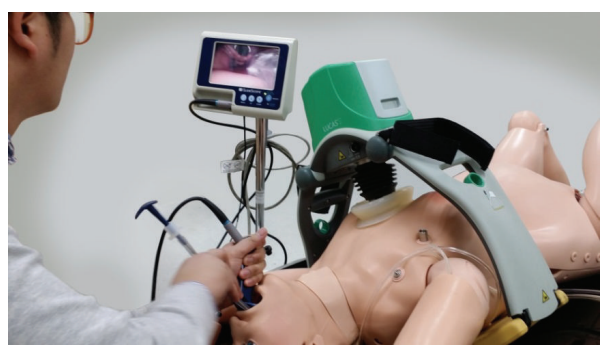

(c)

Figure 1: Endotracheal intubation with the Glidescope during mechanical chest compressions by LUCAS on the wedge. (a) High-fidelity manikin (SimMom) applying LUCAS on the $15^{\circ}$ wedge. (b) $30^{\circ}$ left lateral tilt position created by custom-made wedge. (c) Trial scene with Glidescope during mechanical chest compressions in the $30^{\circ}$ wedge.

in the operating room [15-17]. However, only a foam and hard wedge can maintain an angle of $15^{\circ}-30^{\circ}[18,19]$. No study has investigated the optimal laryngoscope to be used for rapid and successful intubation during chest compressions in maternal CPR using a mechanical compression device with an angle of $15^{\circ}$ and $30^{\circ}$ in the LLT position in an emergency room setting.

The aim of this study was to evaluate laryngoscopes for intubation on simulated difficult airways during maternal CPR with a mechanical compression device at angles of $15^{\circ}$ and $30^{\circ}$ in the LLT position in an emergency room setting. We hypothesized that the required time and success rate for intubation would be different based on the type of laryngoscope used in the above-mentioned situation.

\section{Methods}

2.1. Study Design. We conducted a randomized crossover manikin study to examine intubation with three laryngoscopes under two types of tilt during simulated maternal CPR at our university's simulation centre in March, 2014. The local ethics committee approved this study in January 2014 (HYI14-004-1). We registered the study protocol in Clinical Trials before study initiation (Clinicaltrials.gov: NCT02074072).

2.2. Equipment and Materials. Participants intubated the airway with a direct laryngoscope and two video laryngoscopes using an endotracheal tube with an internal diameter of $6.5 \mathrm{~mm}$ (Portex, St. Paul, MN, USA) and the manufacturer stylet. A direct laryngoscope is the Macintosh laryngoscope (MCL), which has a size-4 curved blade with a Satin Slip Stylet (Mallinckrodt Medical, St. Louis, MO, USA). One video laryngoscope option is the Glidescope (GVL), which has a hyperangulated, nonchannelled, standard-size blade (Verathon, Bothell, WA, USA) with a GlideRite Rigid Stylet. Another option is the Pentax-AWS (AWS), which has a channelled, standard blade (Pentax Corporation, Tokyo, Japan). We used a high-fidelity manikin (SimMom, Laerdal, Stavanger, Norway) for chest compressions and airway intubations. The manikin was set with a tongue edema setting and LUCAS 2 (LUCAS Chest Compression System, PhysioControl/Jolife AB, Lund, Sweden) for performing chest compressions at a continuous rate of 100 compressions/min at a depth of $5 \mathrm{~cm}$, according to guidelines [1]. We used $15^{\circ}$ and $30^{\circ}$ custom-made wedges to simulate the LLT position $(1000 \times$ $600 \times 156 \mathrm{~mm}$ for the $15^{\circ}$ wedge and $1000 \times 600 \times 300 \mathrm{~mm}$ for the $30^{\circ}$ wedge). The manikin was laid on a backboard $(450 \times 600 \times 10 \mathrm{~mm}, 3 \mathrm{~kg}$ Lifeline Plastic, Sung Shim Medical Co., Bucheon, Korea) and placed on the wedge on a bed (Transport stretcher, $760 \times 2110 \mathrm{~mm}, 228 \mathrm{~kg}$, Stryker Co., Kalamazoo, Michigan, US) (Figure 1).

2.3. Participants. The sample size was calculated based on a previous study regarding the time required for intubation during chest compressions with a $27^{\circ}$ LLT [7]. The mean (SD) time was MCL 18.9(4) and AWS 12.6(1.2) to ventilate the lungs after tracheal intubation. To detect a $33 \%$ difference in intubation time with a power of 0.8 , we estimated that 14 operators would be adequate for each device with a $20 \%$ drop rate. We recruited physicians working at one tertiary medical centre in March, 2014. We included healthy volunteers who were between 16 and 60 years old and had more than 50 experiences of intubation using MCL [20]. We excluded people 


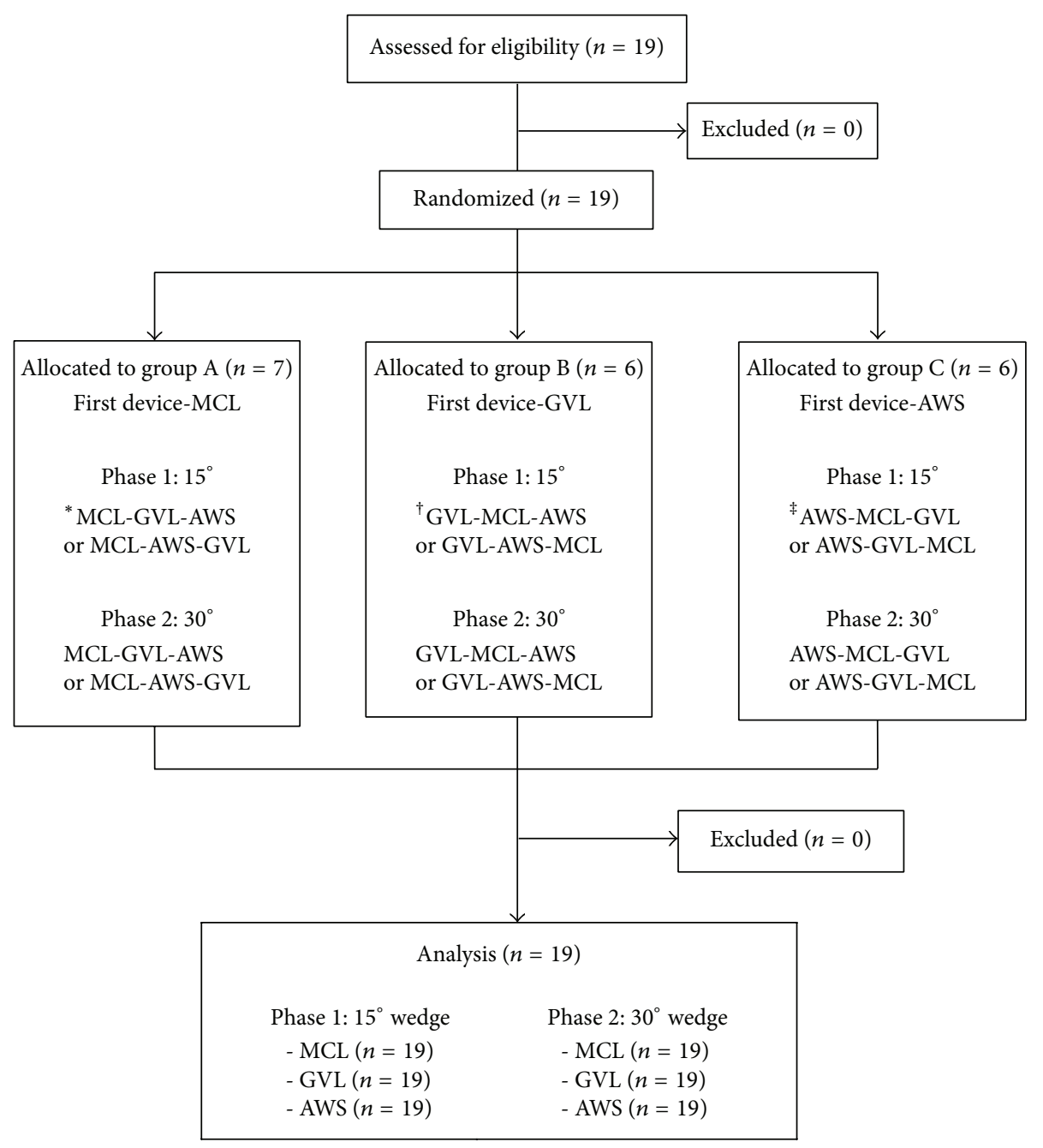

Figure 2: Diagram showing the flow of participants through the study. ${ }^{*} \mathrm{MCL}=\mathrm{Macintosh}$ laryngoscope; ${ }^{\dagger} \mathrm{GVL}=\mathrm{Glidescope} ;{ }^{\ddagger} \mathrm{AWS}=$ Pentax-AWS.

who had wrist and low back disease. All participants signed a written consent form before being included.

2.4. Interventions. All participants completed a brief questionnaire consisting of demographic information (age, gender, body weight, and height) and prior experiences of intubations and maternal CPR in a clinical situation. Ten minutes prior to starting the trials, participants were allowed to practice intubations with all laryngoscopes to familiarize themselves with the Laerdal Airway Management Trainer (Laerdal, Stavanger, Norway) without chest compressions and tongue edema under supine position. Nineteen participants were enrolled, and they were randomly allocated to three groups (http://www.random.org/). Participants in group A $(n=7)$ performed the first intubation with MCL during chest compressions under simulated $15^{\circ}$ and $30^{\circ}$ LLT positions, whereas participants in group $\mathrm{B}(n=6)$ performed the first intubation with GVL. Group C $(n=6)$ performed the first intubation with AWS. After being allocated to the three groups, the participants were placed in a random order by a computer-generated list of random numbers (http://www.random.org/) to minimize learning effects, and then they performed intubation with the laryngoscopes (Figure 2). For MCL, the manikin's head and neck were placed in a sniffing position utilizing several rolled sheets. When each participant performed intubation with each laryngoscope, continuous chest compressions were performed by LUCAS 2. The height of the bed was $80 \mathrm{~cm}$, which was approximately the height of the participant's mid-thigh level. Participants had a 10-minute break after each intubation in one LLT position and a 30-minute break before.

2.5. Outcomes. Primary outcomes were the intubation time and the success rate for intubation. The intubation time was recorded from the start-point to the mid-point and from the mid-point to the endpoint by a recorder. The recorder was informed about how to record the intubation time and was blinded to the objective of this study. The start-point was when the participant inserted the blade between the teeth after command to start by a recorder. The mid-point was when the participant exposed the vocal cord and stated "I can see." The endpoint was at the first manual ventilation 
after intubation, regardless of success or failure of air inflating into the manikin's lungs. The time to visualize the glottis view (TTV) was measured from the start-point to the mid-point, and the time to progress the endotracheal tube (TTP) was consecutively measured from the mid-point to the endpoint. The time to intubate (TTI) was calculated from the start-point to the endpoint (TTV + TTP). We defined intubation failure as follows: when the tip of the tube is not properly placed in the trachea but is placed in the oesophagus or in the oral cavity or when the TTI is $90 \mathrm{sec}$ or more [21, 22].

Secondary outcomes were the glottic view using a Cormack-Lehane score (CLS) and the preference for laryngoscopes. The preference for laryngoscopes was determined by asking the participants to choose the laryngoscope that would be most favourable during maternal CPR.

2.6. Statistical Analysis. The data were compiled using a standard spreadsheet application (Excel, Microsoft, Redmond, WA, USA) and were analysed using the Statistical Package for the Social Sciences (SPSS) 18.0 KO for Windows (SPSS Inc., Chicago, IL, USA). We generated the descriptive statistics and presented them as frequencies and percentages for the categorical data and medians with interquartile ranges (IQR) for the continuous data because the data were not normally distributed. To compare the intubation time among the three laryngoscopes, the Kruskall-Wallis test was used for continuous variables. A $\chi^{2}$ test was used to compare the categorical variables, such as the success rate for intubation, the CLS, and the laryngoscope preference. A post hoc analysis was conducted with the Mann-Whitney test using a Bonferroni correction. $P<0.05$ was considered significant. The KaplanMeier analysis was performed to analyse the cumulative success rate regarding TTV and TTP.

\section{Results}

3.1. General Characteristics. Nineteen participants were enrolled. Nineteen intubation trials were performed for each laryngoscope for both $15^{\circ}$ and $30^{\circ} \mathrm{LLT}$. There was no exclusion in our study. The general characteristics of the participants are shown in Table 1.

3.2. Tracheal Intubation in $15^{\circ}$ LLT Position. TTI was significantly different among the three laryngoscopes. The TTI in AWS was shortest, followed by GVL and MCL (all $P<0.05$ ). The TTV in both GVL and AWS was significantly less than that of MCL, whereas there was no significant difference between the two video laryngoscopes $(P=1.00)$. In terms of TTP, progression of ETT using AWS was faster than that of MCL and GVL (all $P<0.001$ ). However, there was no significant difference between MCL and GVL $(P=0.56)$ (Table 2).

Intubation using AWS showed the highest success rate, followed by GVL and MCL. The glottis views in the two video laryngoscopes were better than that of MCL.

3.3. Tracheal Intubation in $30^{\circ}$ LLT Positions. The TTI in AWS was faster than that of MCL and GVL (all $P<0.05$ ). However, there was no significant difference between MCL and GVL $(P=0.08)$. The TTV in GVL and AWS was significantly lower
TABle 1: Demographic characteristics.

\begin{tabular}{lc}
\hline Characteristics & Data \\
\hline Sex (percent) & Male $(100)$ \\
Age (years) & $33(29-36)$ \\
Height $(\mathrm{cm})$ & $172(171-174)$ \\
Weight $(\mathrm{kg})$ & $78(75-80)$ \\
BMI $\left(\mathrm{kg} / \mathrm{m}^{2}\right)$ & $25.9(24.2-26.6)$ \\
Participants & \\
PGY2 & \\
PGY3 & $3(15.8)$ \\
PGY4 & $3(15.8)$ \\
EP & \\
Intubation experiences & $2(10.5)$ \\
MCL $>50$ (times) & $11(57.9)$ \\
GVL $>50$ (times) & \\
AWS $>50$ (times) & $19(100)$ \\
Clinical experiences & $18(94.7)$ \\
Maternal CPR & $5(26.3)$ \\
Maternal intubation & \\
Intubation during maternal CPR & $9(47.4)$ \\
\hline
\end{tabular}

Categorical variables are given as numbers (percentage). Continuous variables are given as median (IQR). ${ }^{*} \mathrm{PGY}$ : postgraduate years; ${ }^{\dagger} \mathrm{EP}$ : emergency physician.

than that of MCL, whereas there was no significant difference between the two video laryngoscopes $(P=0.71)$. In terms of TTP, progression of ETT using AWS was faster than that of MCL and GVL $(P=0.002, P<0.001$, resp.). However, there was no significant difference between MCL and GVL $(P=0.55)$ (Table 3$)$.

The cumulative success rates related to TTV for AWS and GVL were significantly higher than that of MCL $(P<0.001)$. The cumulative success rate related to TTP for AWS was significantly higher than that of GVL and MCL $(P<0.001)$ (Figure 3).

3.4. Preference for Laryngoscopes. Regardless of tilt degree, 13 participants (68.4\%) preferred AWS, and six participants (31.6\%) selected GVL when asked which of the three laryngoscopes they prefer for maternal CPR.

\section{Discussion}

Pregnancy can be a potent contributor to ventilation difficulty because of edema of the upper airway tract, and motion limitation of the thorax and diaphragm may occur unexpectedly [9-11]. Supraglottic airway devices might be useful to protect the airway and provide ventilation during pregnancy. However, it is known that aspiration is a high risk factor for pregnant women [1]. Compared to an endotracheal tube (ETT), supraglottic airway devices are not enough to prevent aspiration. Therefore, emergent endotracheal intubation in pregnant women deserves special emphasis $[1,11]$.

It can be difficult to perform intubation during CPR because the chest compressions can cause the glottis to move up and down, which makes it hard to maintain proper glottis 
TABLE 2: Tracheal intubation in 15-degree left lateral tilt positions $(n=19)$.

\begin{tabular}{|c|c|c|c|c|c|c|c|}
\hline & \multicolumn{7}{|c|}{15 degrees } \\
\hline & $\begin{array}{c}\text { MCL } \\
(n=19)\end{array}$ & $\begin{array}{c}\text { GVL } \\
(n=19)\end{array}$ & $\begin{array}{c}\text { AWS } \\
(n=19)\end{array}$ & $P$ value & MCL versus AWS & MAC versus GVL & AWS versus GVL \\
\hline \multicolumn{8}{|l|}{$\begin{array}{l}\text { Intubation time } \\
\text { (seconds) }\end{array}$} \\
\hline TTI* $^{*}$ & $\begin{array}{c}36.9 \\
(31.5-50.7)\end{array}$ & $\begin{array}{c}22.9 \\
(17.5-33)\end{array}$ & $\begin{array}{c}12.8 \\
(11.3-15.2)\end{array}$ & $<0.001$ & $<0.001$ & 0.04 & $<0.001$ \\
\hline $\mathrm{TTV}^{\dagger}$ & $\begin{array}{c}16.5 \\
(12.8-26.3)\end{array}$ & $\begin{array}{c}4.9 \\
(4.2-13.5)\end{array}$ & $\begin{array}{c}5.8 \\
(4-7.6)\end{array}$ & $<0.001$ & $<0.001$ & $<0.001$ & 1.00 \\
\hline $\mathrm{TTP}^{\ddagger}$ & $\begin{array}{c}18 \\
(13.8-28)\end{array}$ & $\begin{array}{c}16.2 \\
(12.7-23.2)\end{array}$ & $\begin{array}{c}7.0 \\
(5.3-9.2)\end{array}$ & $<0.001$ & $<0.001$ & 0.56 & $<0.001$ \\
\hline \multicolumn{8}{|l|}{$\begin{array}{l}\text { Success rate } \\
\text { (percent) }\end{array}$} \\
\hline Success rate & $14(73.7)$ & $18(94.7)$ & $19(100)$ & 0.04 & & & \\
\hline \multicolumn{8}{|l|}{ Failure } \\
\hline Esophageal intubation & $3(15.8)$ & $0(0)$ & $0(0)$ & 0.09 & & & \\
\hline Intubation time $>90 \mathrm{~s}$ & $2(10.5)$ & $1(5.3)$ & $0(0)$ & 0.77 & & & \\
\hline \multicolumn{8}{|l|}{ Cormack and Lehane score } \\
\hline I-II & $7(36.8)$ & $17(89.5)$ & $19(100)$ & $<0.001$ & & & \\
\hline III-IV & $12(63.2)$ & $2(10.5)$ & $0(0)$ & & & & \\
\hline Preference & $0(0)$ & $6(31.6)$ & $13(68.4)$ & 1.00 & & & \\
\hline
\end{tabular}

Categorical variables are given as numbers (percentage). Continuous variables are given as median (IQR). MCL: Macintosh laryngoscope; AWS: Pentax-AWS; GVL: Glidescope. ${ }^{*}$ Total time for tracheal intubation. ${ }^{\dagger}$ Time to visualize glottis view. ${ }^{\ddagger}$ Time to progress the tracheal tube from exposure of vocal cords to first ventilation.

TABLE 3: Tracheal intubation in 30-degree left lateral tilt positions $(n=19)$.

\begin{tabular}{|c|c|c|c|c|c|c|c|}
\hline & \multicolumn{7}{|c|}{30 degrees } \\
\hline & $\begin{array}{c}\text { MCL } \\
(n=19)\end{array}$ & $\begin{array}{c}\text { GVL } \\
(n=19)\end{array}$ & $\begin{array}{c}\text { AWS } \\
(n=19)\end{array}$ & $P$ value & MCL versus AWS & MCL versus GVL & AWS versus GVL \\
\hline \multicolumn{8}{|l|}{$\begin{array}{l}\text { Intubation time } \\
\text { (seconds) }\end{array}$} \\
\hline $\mathrm{TTI}^{*}$ & $\begin{array}{c}32.4 \\
(22.6-47.1)\end{array}$ & $\begin{array}{c}21 \\
(16.2-31.2)\end{array}$ & $\begin{array}{c}13.4 \\
(12.4-16.2)\end{array}$ & $<0.001$ & $<0.001$ & 0.09 & 0.001 \\
\hline $\mathrm{TTV}^{\dagger}$ & $\begin{array}{c}13.9 \\
(9-23.9)\end{array}$ & $\begin{array}{c}5.2 \\
(4.0-8.9)\end{array}$ & $\begin{array}{c}5.4 \\
(4.7-8.2)\end{array}$ & $<0.001$ & $<0.001$ & 0.004 & 0.71 \\
\hline $\mathrm{TTP}^{\ddagger}$ & $\begin{array}{c}16.2 \\
(13.6-21.8)\end{array}$ & $\begin{array}{c}14.6 \\
(12.3-18.6)\end{array}$ & $\begin{array}{c}7.5 \\
(6.3-9.9)\end{array}$ & 0.002 & 0.002 & 0.55 & $<0.001$ \\
\hline \multicolumn{8}{|l|}{$\begin{array}{l}\text { Success rate } \\
\text { (percent) }\end{array}$} \\
\hline Success rate & $9(47.4)$ & $17(89.5)$ & $18(94.7)$ & 0.001 & & & \\
\hline \multicolumn{8}{|l|}{ Failure } \\
\hline Esophageal intubation & $4(21.1)$ & $0(0)$ & $1(5.3)$ & 0.11 & & & \\
\hline Intubation time $>90 \mathrm{~s}$ & $7(36.8)$ & $2(10.5)$ & $0(0)$ & 0.007 & & & \\
\hline \multicolumn{8}{|l|}{ Cormack-Lehane score } \\
\hline I-II & $5(26.3)$ & $18(94.7)$ & $19(100)$ & $<0.001$ & & & \\
\hline III-IV & $14(73.7)$ & $1(5.3)$ & $0(0)$ & & & & \\
\hline Preference & $0(0)$ & $6(31.6)$ & $13(68.4)$ & 1.00 & & & \\
\hline
\end{tabular}

Categorical variables are given as numbers (percentage). Continuous variables are given as median (IQR). MCL: Macintosh laryngoscope; AWS: Pentax-AWS; GLS: Glidescope. ${ }^{*}$ Total time for tracheal intubation. ${ }^{\dagger}$ Time to visualize glottis view. ${ }^{*}$ Time to progress the tracheal tube from exposure of vocal cords to first ventilation. 


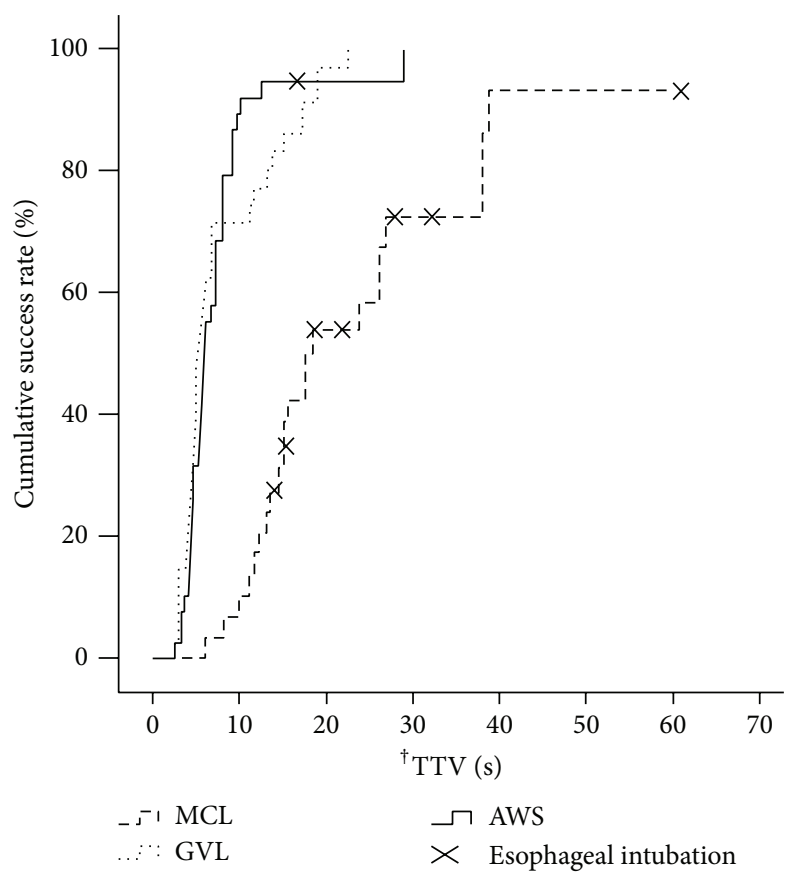

(a)

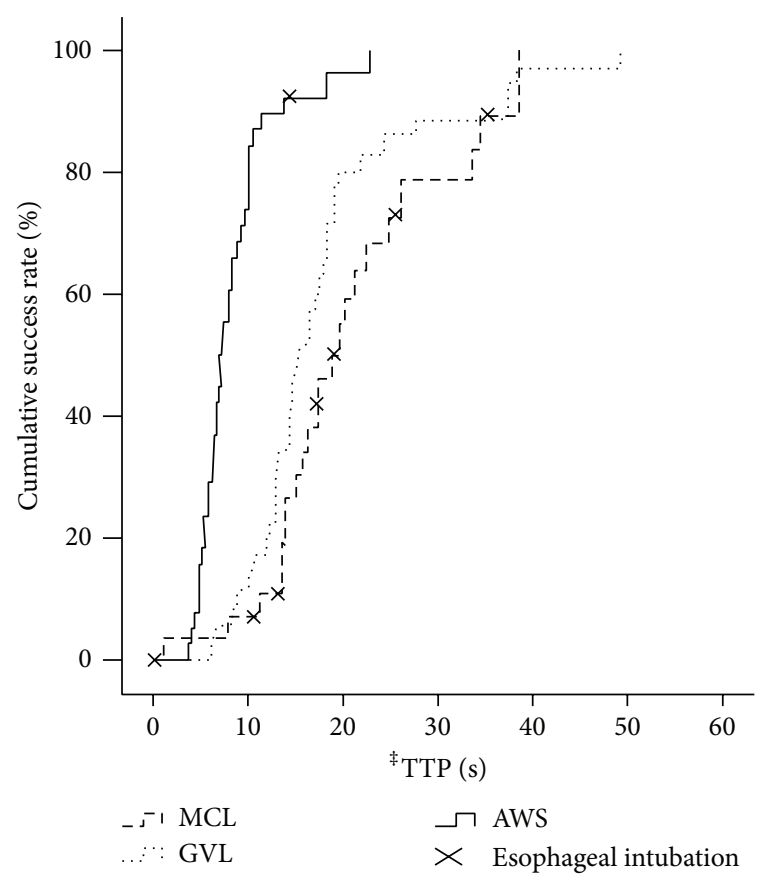

(b)

FIGURE 3: Cumulative success rate related to (a) the time from blade insertion to exposure of the vocal cords (TTV) and (b) the time from exposure of the vocal cords to manual ventilation (TTP) in the left lateral tilt position with mechanical chest compressions. MCL: Macintosh laryngoscope; AWS: Pentax-AWS; GVL: Glidescope; ${ }^{\dagger} \mathrm{TTV}$ : time to visualize glottis view; ${ }^{\ddagger} \mathrm{TTV}$ : time to progress the tracheal tube from exposure of vocal cords to first ventilation.

view [7]. Furthermore, because patients are placed in the LLT position during maternal CPR, the lifting force of the intubator to expose the glottis view can be insufficiently transmitted to patients. It is also difficult to handle the laryngoscope with patients in the LLT position [7]. Even if the glottis is fully exposed, chest compressions and the LLT position can interrupt intubators in the progression of the ETT and removal of the stylet [7]. Therefore, the TTI can be affected by the degree of tilt, chest compression, and the type of laryngoscope. Kohama et al. reported TTI in AWS during simulated maternal CPR (normal airway setting, manual chest compression, and $27^{\circ}$ LLT position) using an operating table that was shorter than that of MCL [7]. In this study, we evaluated the TTI for laryngoscopes in different settings (tongue edema setting, mechanical chest compression, and two types of LLT positions using $15^{\circ}$ and $30^{\circ}$ wedges) on a hospital bed in an emergency room and compared it to previous studies. We found that the use of AWS for emergent intubation reduced the TTV and TTP, which resulted in the reduction of the TTI compared with that of MCL at both tilt degrees. The use of GVL significantly decreased the TTV but hardly reduced the TTP, which also resulted in a reduction of the TTI compared with that of MCL. This means that the use of a camera attached to the blade of a video laryngoscope may play a major role in decreasing the time to exposure of the vocal cords. In terms of TTP, AWS showed better outcomes than GVL when compared with MCL. It is believed that the channelled blade applied to AWS enables the ETT to progress effectively [4]. Because AWS does not require a stylet, AWS can also reduce the time required to handle a stylet in GVL during maternal CPR. AWS was the most preferred device among the three laryngoscopes in this study, which we assume is due to the integrated channel of the blade that enables faster tracheal intubation without a stylet.

The ERC guideline recommends a $15^{\circ}-30^{\circ}$ LLT position to remove the caval compression during maternal CPR [1]. Two studies have reported that the TTI was affected by the patient position and the type of laryngoscope used. When using MCL, the TTI in the supine position was shorter than that in the LLT position [8]. However, in terms of AWS, there was no difference in the TTI between these positions [8]. In this study, participants performed intubations at two tilt angles $\left(15^{\circ}\right.$ and $\left.30^{\circ}\right)$, excluding the supine position. Angles of $15^{\circ}$ and $30^{\circ}$ are the minimum and maximum tilt angles, respectively, that are recommended by the ERC guideline for maternal CPR [1]. There were no differences in the TTI between the tilt angles for the three laryngoscopes.

Regarding the success rate for intubation, previous studies have reported that this is affected by chest compressions and the type of laryngoscope used [7]. In the $27^{\circ}$ LLT position, MCL showed a lower success rate with chest compressions than without chest compressions [7]. However, AWS showed an equal success rate regardless of chest compression [7]. In the supine position during chest compressions, MCL showed a lower success rate than AWS and GVL $[4,23]$. In this study, we evaluated intubation performance in the $15^{\circ}$ and $30^{\circ}$ LLT 
positions during chest compression. AWS and GVL showed better success rates than MCL at both tilt degrees, as expected. The cumulative success rates had the same results.

\section{Limitation}

There were several limitations of this study.

We used a high-fidelity manikin setting with tongue edema to simulate a difficult airway during pregnancy. This manikin can also simulate difficult airway applying trismus setting, however, which has not been known as typical change of maternal airway. However, the actual maternal airway cannot be simulated by only tongue edema. Furthermore, there could be various clinical anatomic changes due to pregnancy in addition to tongue edema. Therefore, the measured intubation performance in this study could differ from that of actual maternal CPR. Further studies using more sophisticated manikin or clinical trials would be needed.

Recently, mechanical chest compression devices have been widely used, and some studies have reported that mechanical chest compression devices could show better outcomes than manual chest compressions [3]. We used LUCAS Chest Compression System (Physio-Control/Jolife AB, Lund, Sweden) for even and uninterrupted chest compressions. The effect of mechanical chest compressions on performing intubation during chest compressions might differ from that of manual chest compressions.

The most appropriate bed height for chest compressions is different from that of intubation. To best perform chest compressions, the bed height needs to be at lower- to midthigh level $[24,25]$. In contrast, the bed height has to be raised to sternum level to perform intubation well. When intubation is performed using MCL, intubation performance could be influenced by the height of the bed. This is because the sightline view of the intubator is equated to the glottic opening to successfully intubate using MCL. However, in this study, the bed height was fixed at $80 \mathrm{~cm}$, which was approximately mid-thigh level for the participants performing chest compressions. Therefore, the effects of bed height changes on intubation performance were not reflected in the study.

The pressures generated by laryngoscopes can cause deleterious effects to the soft tissues of the upper airway. If capillaries of airway soft tissue are engorged due to pregnancy, it can easily bleed under the pressure generated by laryngoscopes. In previous study, intubation using GVL could reduce the forces to the soft tissues of upper airway when compared to that of MCL [26]. Likewise, video laryngoscopes are considered to be advantageous to lower the pressure to the soft tissue of upper airway in maternal airway management. However, the pressures generated by laryngoscopes are not considered in this study.

\section{Conclusions}

The AWS could be an appropriate laryngoscope for airway management of pregnant women in tilt CPR considering intubation time and success rate.

\section{Conflict of Interests}

Hyunggoo Kang, as the corresponding author of this paper, declares that there is no conflict of interests related to this paper.

\section{Authors' Contribution}

Sanghyun Lee and Wonhee Kim contributed equally to this study. Sanghyun Lee and Wonhee Kim were involved in all aspects of the study design, designing and managing the study, interpreting findings, and cowriting the paper. Hyunggoo Kang, Yoonjae Lee, and Jun Hwi Cho were involved in critical revision of the paper for important intellectual content. Tae Ho Lim, Jaehoon Oh, and Changsun Kim were involved in study concept and design, interpretation of the data, critical revision of the paper for important intellectual content, and final approval of the version to be published.

\section{Acknowledgment}

The research fund of Hanyang University (201400000000505) supported this paper.

\section{References}

[1] J. Soar, G. D. Perkins, G. Abbas et al., "European Resuscitation Council Guidelines for Resuscitation 2010 Section 8. Cardiac arrest in special circumstances: electrolyte abnormalities, poisoning, drowning, accidental hypothermia, hyperthermia, asthma, anaphylaxis, cardiac surgery, trauma, pregnancy, electrocution," Resuscitation, vol. 81, no. 10, pp. 1400-1433, 2010.

[2] S. Kim, J. S. You, H. S. Lee et al., "Quality of chest compressions performed by inexperienced rescuers in simulated cardiac arrest associated with pregnancy," Resuscitation, vol. 84, no. 1, pp. 98-102, 2013.

[3] M. Westfall, S. Krantz, C. Mullin, and C. Kaufman, "Mechanical versus manual chest compressions in out-of-hospital cardiac arrest: a meta-analysis," Critical Care Medicine, vol. 41, no. 7, pp. 1782-1789, 2013.

[4] S. K. Han, D. H. Shin, and P. C. Choi, "Utility of the PentaxAWS without interruption of chest compression: comparison of the Macintosh laryngoscope with the Pentax-AWS in manikin model," Resuscitation, vol. 81, no. 1, pp. 69-73, 2010.

[5] D. H. Shin, P. C. Choi, and S. K. Han, "Tracheal intubation during chest compressions using Pentax-AWS, GlideScope, and Macintosh laryngoscope: a randomized crossover trial using a mannequin," Canadian Journal of Anesthesia, vol. 58, no. 8, pp. 733-739, 2011.

[6] N. Komasawa, R. Ueki, M. Itani, S.-I. Nishi, and Y. Kaminoh, "Validation of the Pentax-AWS airwayscope utility as an intubation device during cardiopulmonary resuscitation on the ground," Journal of Anesthesia, vol. 24, no. 4, pp. 582-586, 2010.

[7] H. Kohama, N. Komasawa, R. Ueki et al., "Utility of the PentaxAWS airwayscope and macintosh laryngoscope for airway management during chest compressions in 27 left-lateral tilt: a manikin simulation study of maternal cardiopulmonary resuscitation," Journal of Anesthesia, vol. 27, no. 5, pp. 671-675, 2013.

[8] N. Komasawa, R. Ueki, M. Itani, H. Nomura, S.-I. Nishi, and Y. Kaminoh, "Evaluation of tracheal intubation in several positions by the Pentax-AWS airway scope: a manikin study," Journal of Anesthesia, vol. 24, no. 6, pp. 908-912, 2010. 
[9] M. S. Suresh, C. L. Mason, and U. Munnur, "Cardiopulmonary resuscitation and the parturient," Best Practice \& Research: Clinical Obstetrics \& Gynaecology, vol. 24, no. 3, pp. 383-400, 2010.

[10] S. E. King and D. A. Gabbott, "Maternal cardiac arrest-rarely occurs, rarely researched," Resuscitation, vol. 82, no. 7, pp. 795796, 2011.

[11] T. L. Vanden Hoek, L. J. Morrison, M. Shuster et al., "Part 12: cardiac arrest in special situations: 2010 American Heart Association guidelines for cardiopulmonary resuscitation and emergency cardiovascular care," Circulation, vol. 122, pp. S829S861, 2010.

[12] H. Finegold, C. A. Troianos, and H. Basi, "Use of advanced airway techniques in the pregnant patient," Anesthesiology Clinics, vol. 31, no. 3, pp. 529-543, 2013.

[13] H. Lombaard, P. Soma-Pillay, and E.-M. Farrell, "Managing acute collapse in pregnant women," Best Practice \& Research: Clinical Obstetrics \& Gynaecology, vol. 23, no. 3, pp. 339-355, 2009.

[14] F. M. Jeejeebhoy and L. J. Morrison, "Maternal cardiac arrest: a practical and comprehensive review," Emergency Medicine International, vol. 2013, Article ID 274814, 8 pages, 2013.

[15] D. A. Gabbott, "Uterine displacement during CPR in the pregnant patient-why bother?" Resuscitation, vol. 84, no. 3, pp. 267-268, 2013.

[16] S. S. Lipman, J. Y. Wong, J. Arafeh, S. E. Cohen, and B. Carvalho, "Transport decreases the quality of cardiopulmonary resuscitation during simulated maternal cardiac arrest," Anesthesia \& Analgesia, vol. 116, no. 1, pp. 162-167, 2013.

[17] S. Lipman, K. Daniels, S. E. Cohen, and B. Carvalho, "Labor room setting compared with the operating room for simulated perimortem cesarean delivery: a randomized controlled trial," Obstetrics \& Gynecology, vol. 118, no. 5, pp. 1090-1094, 2011.

[18] J. K. Ip, J. P. Campbell, D. Bushby, and S. M. Yentis, "Cardiopulmonary resuscitation in the pregnant patient: a manikin-based evaluation of methods for producing lateral tilt," Anaesthesia, vol. 68, no. 7, pp. 694-699, 2013.

[19] M. Butcher, J. Ip, D. Bushby, and S. M. Yentis, "Efficacy of cardiopulmonary resuscitation in the supine position with manual displacement of the uterus vs lateral tilt using a firm wedge: a manikin study," Anaesthesia, vol. 69, no. 8, pp. 868871, 2014.

[20] S. M. Je, Y. C. Cho, H. J. Choi, B. Kang, T. Lim, and H. Kang, "An application of the learning curve-cumulative summation test to evaluate training for endotracheal intubation in emergency medicine," Emergency Medicine Journal, 2013.

[21] J. L. Benumof, R. Dagg, and R. Benumof, "Critical hemoglobin desaturation will occur before return to an unparalyzed state following $1 \mathrm{mg} / \mathrm{kg}$ intravenous succinylcholine," Anesthesiology, vol. 87, no. 4, pp. 979-982, 1997.

[22] S. D. Weingart and R. M. Levitan, "Preoxygenation and prevention of desaturation during emergency airway management," Annals of Emergency Medicine, vol. 59, no. 3, pp. 165-175, 2012.

[23] T. Xanthos, K. Stroumpoulis, E. Bassiakou et al., "Glidescope videolaryngoscope improves intubation success rate in cardiac arrest scenarios without chest compressions interruption: a randomized cross-over manikin study," Resuscitation, vol. 82, no. 4, pp. 464-467, 2011.

[24] A. Lewinsohn, P. B. Sherren, and D. S. Wijayatilake, "The effects of bed height and time on the quality of chest compressions delivered during cardiopulmonary resuscitation: a randomised crossover simulation study," Emergency Medicine Journal, vol. 29, no. 8, pp. 660-663, 2012.

[25] C. K. Hong, S. O. Park, H. H. Jeong et al., "The most effective rescuer's position for cardiopulmonary resuscitation provided to patients on beds: a randomized, controlled, crossover mannequin study," Journal of Emergency Medicine, vol. 46, no. 5, pp. 643-649, 2014.

[26] M. Carassiti, R. Zanzonico, S. Cecchini, S. Silvestri, R. Cataldo, and F. E. Agrò, "Force and pressure distribution using Macintosh and GlideScope laryngoscopes in normal and difficult airways: a manikin study," British Journal of Anaesthesia, vol. 108, no. 1, pp. 146-151, 2012. 


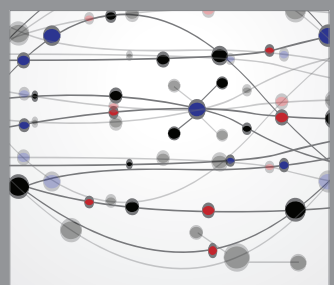

The Scientific World Journal
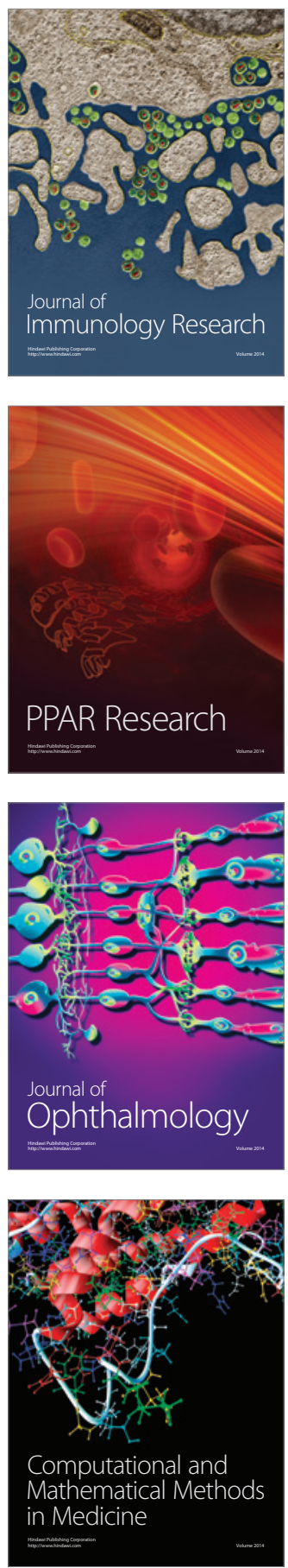

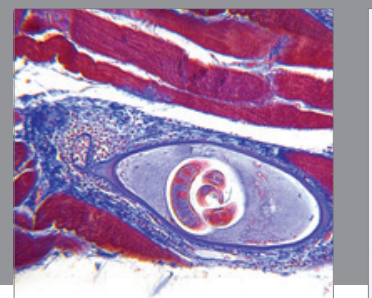

Gastroenterology

Research and Practice
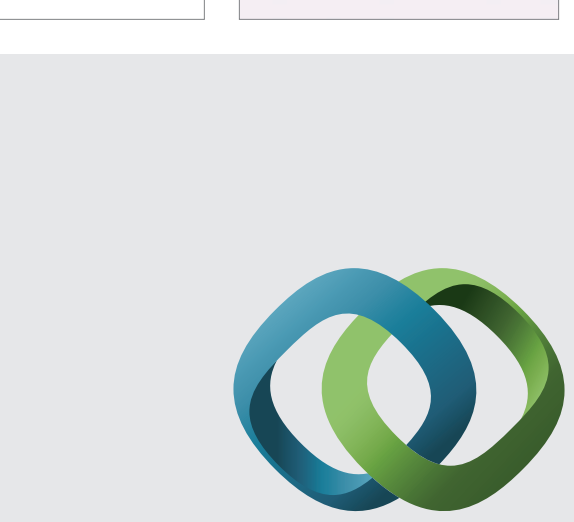

\section{Hindawi}

Submit your manuscripts at

http://www.hindawi.com
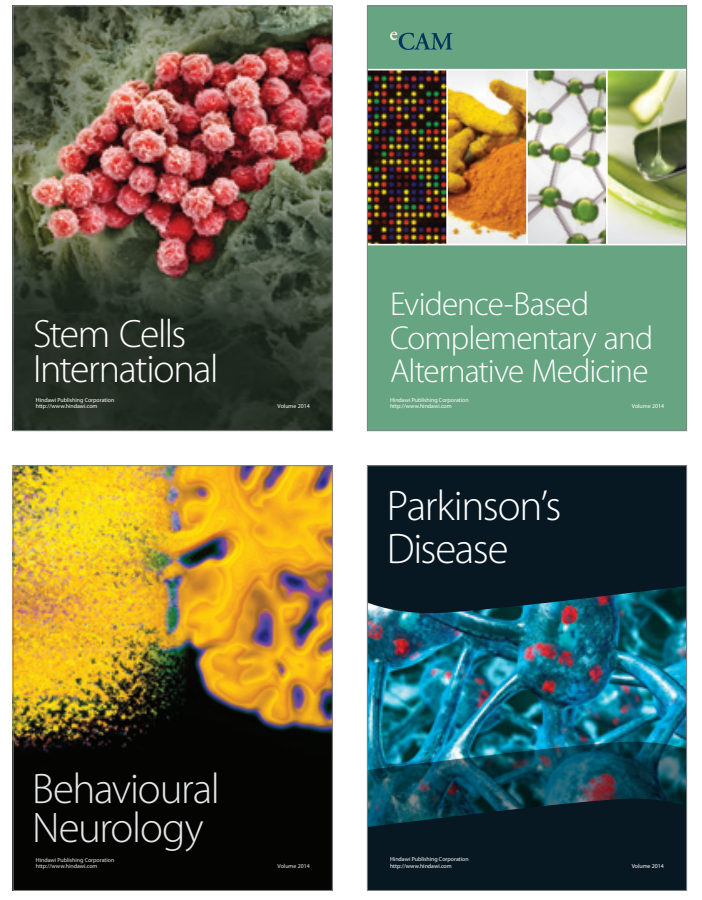
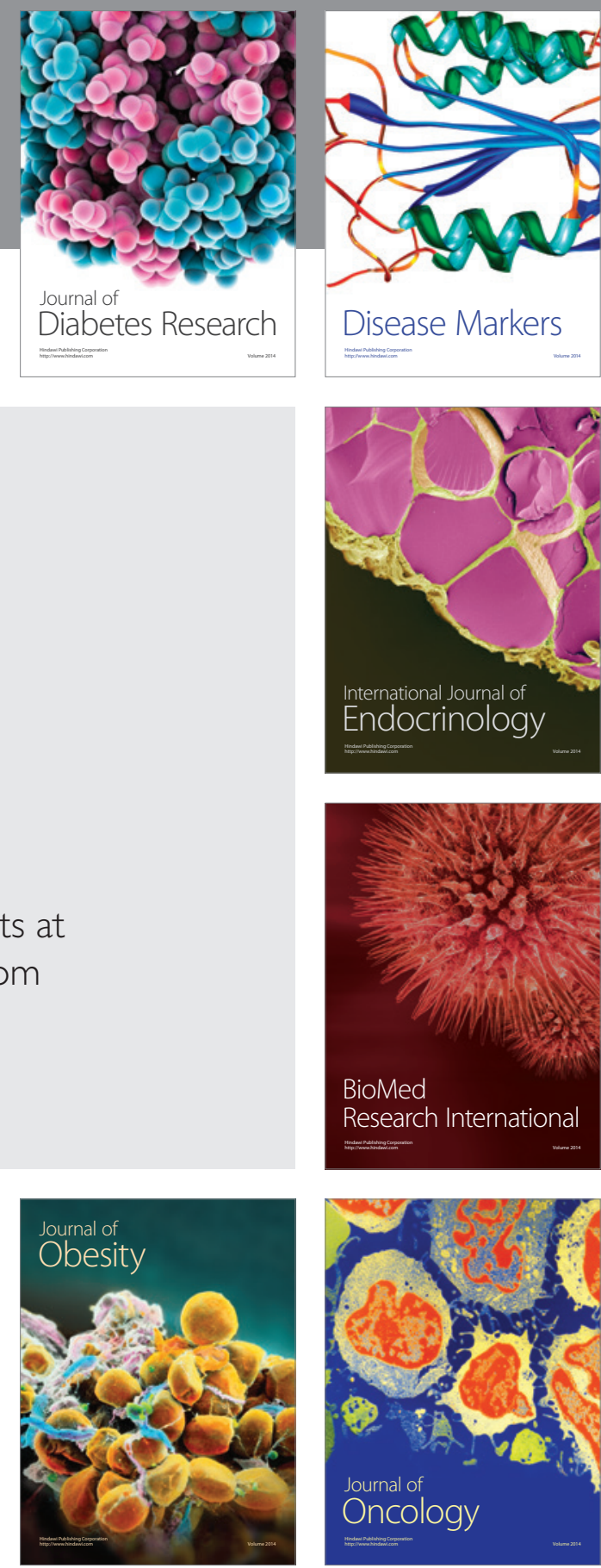

Disease Markers
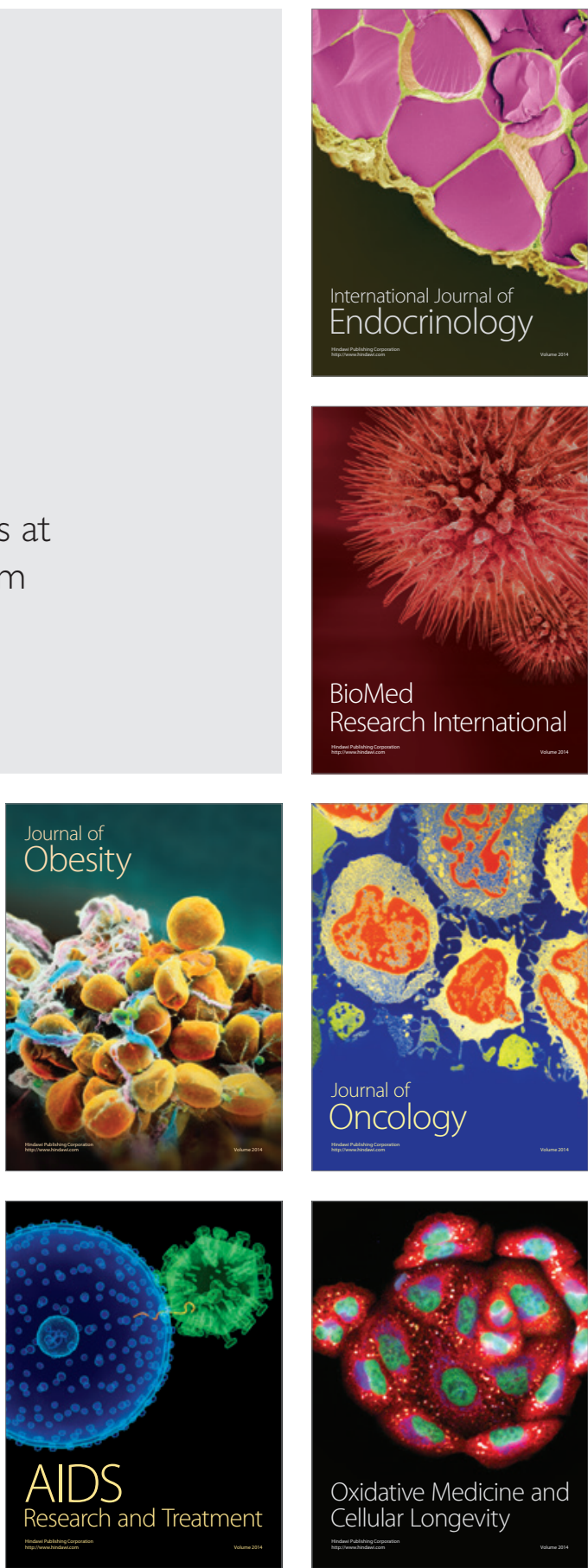